\title{
Renal Dysplasia in a Shih Tzu Dog in Japan
}

\author{
Kiminari OHARA ${ }^{1)}$, Yoshiyasu KOBAYASHI ${ }^{2) *}$, Noriko TSUCHIYA ${ }^{2)}$, Hidefumi FURUOKA ${ }^{2)}$ and Takane MATSUI ${ }^{2)}$ \\ ${ }^{1)}$ Ohara Animal Hospital, 13-32 Yamadahira, Kawada, Shinshiro, Aichi 441-1346 and ${ }^{2)}$ Department of Veterinary Pathology, Obihiro \\ University of Agriculture and Veterinary Medicine, Obihiro 080-8555, Japan
}

(Received 19 March 2001/Accepted 12 July 2001)

ABSTRACT. A 5-month-old, male, Shih Tzu dog manifesting polyuria and polydipsia since 2-month-old was presented to our hospital with additional clinical complaints of vomiting and depression during recent a few days. Despite the symptomatic therapy for chronic renal failure, he died on the day after medication. Macroscopically, both kidneys were small in size with rough surface. Microscopical examination revealed bilateral renal fibrosis with dysplastic changes consisting of immature glomeruli and tubules, and foci of adenomatoid proliferation of tubular epithelium. In addition, incomplete lobulation of medulla with pelvic structures was also noticed in the right kidney. From these findings, the present case was diagnosed as renal dysplasia in Shih Tzu dog which was documented in the literatures. KEY WORDS: canine, renal dysplasia, Shih Tzu.

J. Vet. Med. Sci. 63(10): 1127-1130, 2001

Chronic renal failure or renal fibrosis occurs most frequently in adult or aging domestic animals, particularly in dogs and cats, and may have a number of causes [1]. One of the most common clinical sings observed in this chronic renal disease state is dysfunction to concentrate urine, resulting in polyuria, dehydration and polydipsia [1]. Hypoplastic anemia also occurs as the result of failure to synthesize and secrete erythropoietin in the kidney [1].

The development of severe bilateral renal fibrosis has also been reported in young dogs of several breeds and designated as progressive juvenile nephropathy, canine renal dysplasia or familial renal disease [1-4, 6-8]. There are various degrees of compensatory, degenerative and inflammatory changes in the lesion. Renal dysplasia of Golden Retriever dog has been reported in Japan [5]. However, there is no report describing renal dysplasia in Shih Tzu dogs in Japan. This paper deals with the clinical and pathological characteristics of renal dysplasia in a Shih Tzu dog.

A 5-month-old, male, Shih Tzu dog weighing $4.0 \mathrm{~kg}$ manifesting polyuria and polydipsia since 2-month-old was presented to our hospital with additional clinical complaints of vomiting and depression during recent a few days. Physical examination revealed severe depression and dehydration. The kidneys were not detected in abdominal radiographs. Clinical pathology revealed hypoplastic anemia (PCV: $24 \%$ ), azotemia (blood urea nitrogen: $224.0 \mathrm{mg}$ / $\mathrm{d} l)$, increased creatinine $(12.0 \mathrm{mg} / \mathrm{d} l)$, and hyperkalemia $(6.7 \mathrm{mmol} / l)$. The animal received fluid therapy and glucocorticoid therapy. Despite these symptomatic therapy for chronic renal failure, the animal died on the day after medication.

With request by the owner, partial necropsy restricted to the abdominal cavity was performed. Macroscopically, both kidneys were firm and small in size with rough surface (Fig. 1). On cut surface of the kidneys, the cortico-medul-

\footnotetext{
* Correspondence to: Kobayashi, Y., Department of Veterinary Pathology, Obihiro University of Agriculture and Veterinary Medicine, Obihiro 080-8555, Japan.
}

lary ratio was markedly reduced. The right kidney was larger than the left one with relatively preserved area (Fig. 1). The cortico-medullary junction of the right kidney was irregular. There were no significant lesions in other abdominal organs including ureter and urinary bladder.

Both kidneys were fixed in the $10 \%$ buffered formalin and embedded in paraffin wax by a routine procedure. Paraffin sections were stained with hematoxylin and eosin (HE), Masson's trichrome, and periodic acid-methenaminesilver impregnation.

Histopathological examination revealed bilateral severe renal fibrosis, particularly in the medulla (Fig. 2). The cortex was very thin with fibrosis and contained some hypertrophic glomeruli and urinary tubules. Cystic dilation of Bowman's capsular space and some sclerotic glomeruli were also noted in the cortex. Mineralization of basement membranes of the Bowman's capsules and urinary tubules

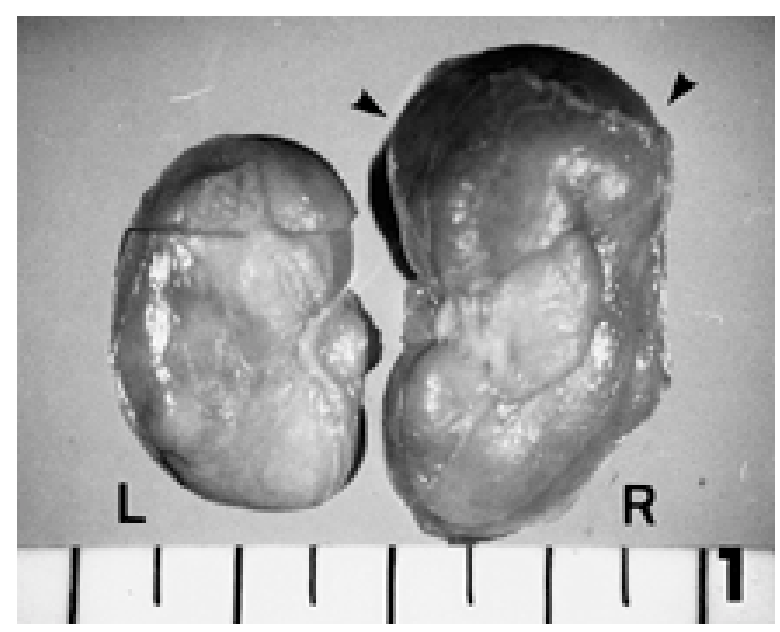

Fig. 1. Both kidneys are small in size with rough surface. The right kidney $(\mathrm{R})$ is larger than the left one (L) with relatively preserved area (arrowheads). 

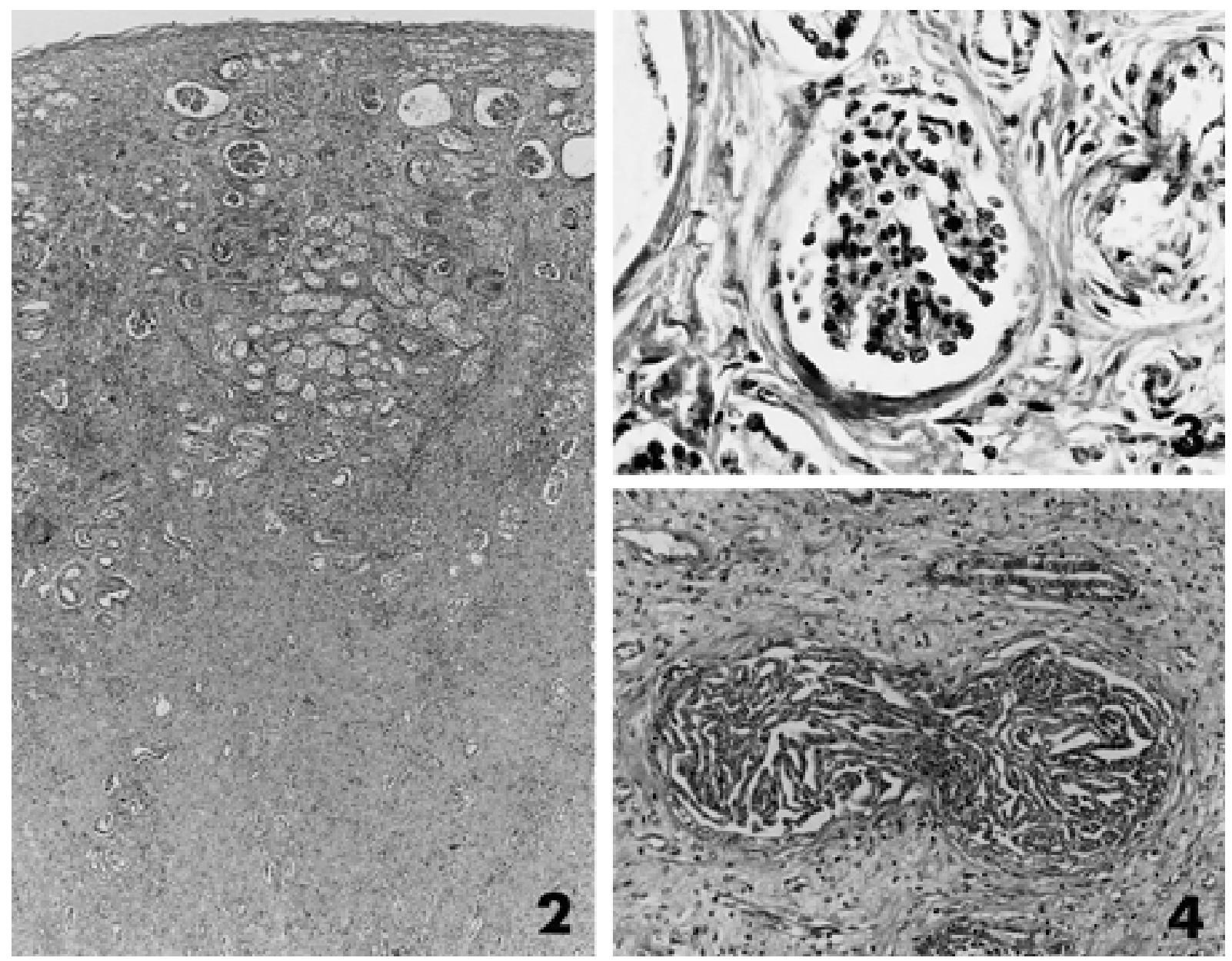

Fig. 2. Left kidney. Severe renal fibrosis, especially in the medulla. Masson's trichrome stain. $\times 44$.

Fig. 3. Left kidney. A immature glomerulus with mineralization of the basement membrane of the Bowman's capsule. Masson's trichrome stain. $\times 396$.

Fig. 4. Left kidney. Adenomatoid proliferation of cuboidal epithelium in a dilated collecting duct. Masson's trichrome stain. $\times 139$.

were frequently observed. In the medulla, there was slight inflammatory cell infiltration, including neutrophils. In addition, there were some immature glomeruli (Fig. 3) and primitive tubules lined by hyperchromatic epithelium in the cortex. Proliferation of arterioles was also seen in the interstitium of the cortex. In the medulla, adenomatoid proliferation of cuboidal epithelium in the dilated lumina of collecting ducts was also noted (Fig. 4). Persistent mesenchyma, which does not stained blue with Masson's trichrome, was not apparent.

In addition to these changes, incomplete lobulation of medulla with pelvic structures was also noticed in the right kidney (Fig. 5). Furthermore, some urinary tubules in deep cortex of the right kidney were irregular in shape and showed increased cellularity of the epithelial lining (Fig. 6). The hypercellular area was composed of stratified epithelial cells with low nucleus/cytoplasm ratio. The margin between severely atrophied and relatively preserved areas in the right kidney was also clearly demonstrated in histopathological examination, and the relatively preserved area showed compensatory hypertrophy of glomeruli and urinary tubules with widespread mineralization (Fig. 7).

Renal dysplasia is defined as disorganized development of renal parenchyma due to abnormal differentiation [4]. In dogs, the lesions associated with dysplasia include fetal/ immature glomeruli and/or tubules, persistent mesenchyma, persistent metanephric ducts, adenomatoid tubular epithelium which lined collecting duct, or dysontogenic metaplasia [7]. Associated with and often obscuring the primary lesions of renal dysplasia, various degrees of compensatory, degenerative and inflammatory lesions are observed in the affected kidneys [7]. In both kidneys of the present case, there were immature glomeruli and tubules, and adenomatoid proliferation of tubular epithelium in the collecting ducts. Thus the present case was pathologically diagnosed as bilateral renal dysplasia with severe fibrosis. 

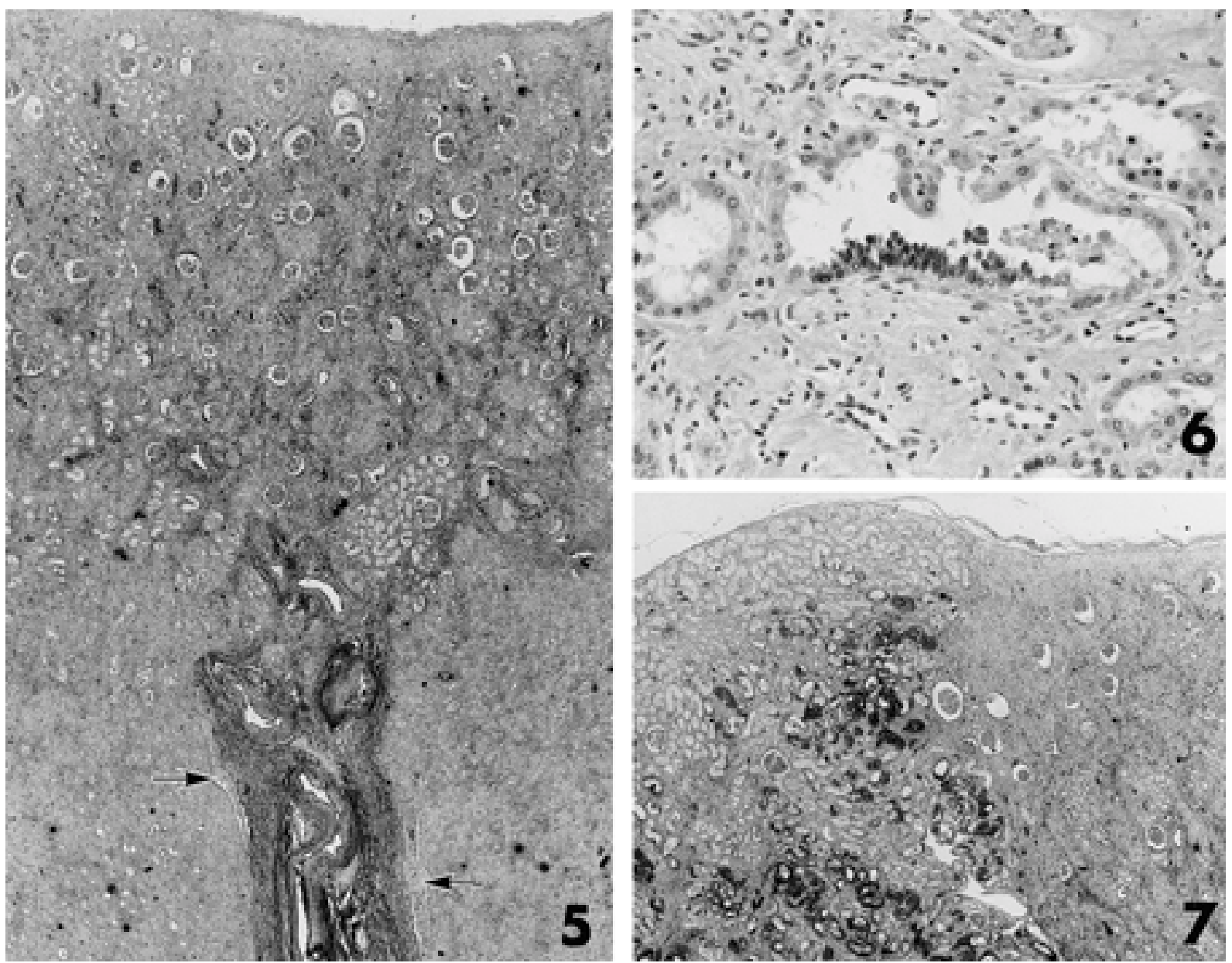

Fig. 5. Right kidney. Marked renal fibrosis with incomplete lobulation of medulla. The medullary structures are separated by renal pelvis (arrows) and connective tissue. HE. $\times 22$.

Fig. 6. Right kidney. A urinary tubule with irregular shape. Note the increased cellularity of the epithelial lining. The hypercellular area is composed of stratified epithelial cells with low nucleus/cytoplasm ratio. HE. $\times 198$.

Fig. 7. Right kidney. Margin between severely fibrosed (right) and relatively preserved (left) areas is clearly demonstrated. Relatively preserved area shows compensatory hypertrophy of glomeruli and urinary tubules with widespread mineralization. HE. $\times 18$.

In addition, incomplete lobulation of medullary structure and urinary tubules with increased cellularity of the epithelial lining were noticed in the right kidney of the present case. The former lesion might reflect a disorganized development of renal parenchyma. However, the significance of the latter proliferative change remains unclear.

In Shih Tzu dogs, renal dysplasia has been reported in the literatures as progressive nephropathy, progressive juvenile nephropathy, or familial nephropathy with autosomal recessive inheritance $[1,2,4,6]$. It has been reported that there are two different clinical manifestations in progressive juvenile nephropathy of Shih Tzu dog [2]. One type is seen in young dogs presenting with only a short history of depression, polydipsia and vomiting and the affected dogs die after a short clinical course. The other type is found to be in good health condition, but blood analysis and urine examination indicate the signs of renal disease. These dogs can live for several years with gradually developing azotemia and anemia. The clinical features of the present case were concurred with those of previous one.

Unfortunately, we could not discuss the familial history. However, clinical and pathological similarities among the progressive nephropathy in Shih Tzu dog and the present case may indicate the possibility of the prevalence of this disease in Japan.

\section{REFERENCES}

1. Confer, A. W. and Panciera R. J. 1995. pp. 209-246. In: Thomson's Special Veterinary Pathology, 2nd ed. (Carlton, W. W. and McGavin, M. D. eds.), Mosby-Year Book, Inc., St. Louis.

2. Hoppe, A., Swenson, L., Jnsson, L. and Hedhammar, A. 1990. 
J. Small Anim. Pract. 31: 83-91.

3. Kerlin, R. L. and Van Winkle, T. J. 1995. Vet. Pathol. 32: 327329.

4. Maxie, M. G. 1993. pp. 447-538. In: Pathology of Domestic Animals, vol. 2, 4th ed. (Jubb, K. V. F., Kennedy, P. C. and Palmer, N. eds.), Academic Press, San Diego.

5. Miyamoto, T., Demura, M., Matsuyama, S., Ohashi, F.,
Fukata, T., Baba, E., Kuwamura, M., Yamate, J., Kotani, T. and Kin, T. 2000. J. Anim. Clin. Med. 9: 137-141 (in Japanese with English abstract).

6. O'brien, T. D., Osborne, C. A., Yano, B. L. and Barnes, D. M. 1982. J. Am. Vet. Med. Assoc. 180: 658-664.

7. Picut, C. A. and Lewis, R. M. 1987. Vet. Pathol. 24: 156-163.

8. Vilafranca, M. and Ferrer, L. 1994. Vet. Pathol. 31: 375-377. 